\title{
Upregulation of IL-10 expression inhibits the proliferation of human periodontal ligament stem cells
}

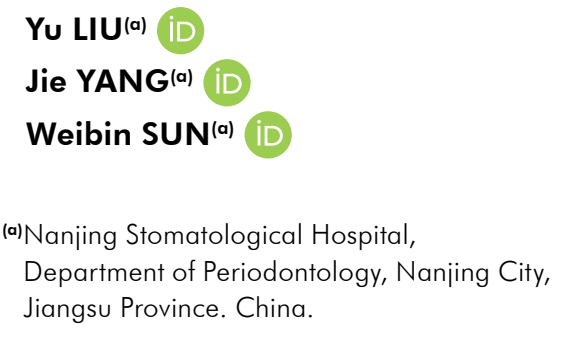

Declaration of Interests: The authors certify that they have no commercial or associative interest that represents a conflict of interest in connection with the manuscript.

\section{Corresponding Author:}

Weibin Sun

E-mail: weibinsun@aliyun.com

https://doi.org/10.1590/1807-3107bor-2020.vol34.0030

Submitted: November 23, 2018

Accepted for publication: September 19, 2019 Last revision: January 6, 2020

\begin{abstract}
The abnormal increase in proliferation rate of human periodontal ligament stem cells (PDLSCs) is considered to be involved in the pathogenesis of periodontitis, a disease in which the IL-10-mediated anti-inflammatory pathway plays a critical role. This study aimed to investigate the involvement of microRNA-4661 in periodontitis and to explore the possible interaction between IL-10 and microRNA-4661. PDLSCs were obtained from periodontitis-affected teeth and healthy control teeth. The expression of microRNA-4661 and IL-10 mRNA was measured in PDLSCs using RT-qPCR. The proliferation ability of PDLSCs was analyzed using CCK-8 assays. Overexpression of microRNA-4661 in a PDLSC cell line was established using two different types of PDLSCs, and the effect of microRNA-466l overexpression on IL-10 expression and cell proliferation were detected by western blot and CCK- 8 assays, respectively. We found that expression levels of microRNA-466l and IL-10 mRNA were significantly lower $(P<0.05)$ in PDLSCs derived from periodontitis-affected teeth compared to those derived from healthy teeth. However, the cell proliferation ability was significantly higher in the PDLSCs derived from periodontitis-affected teeth. Meanwhile microRNA-466l overexpression decreased cell proliferation rates of both types of PDLSCs and upregulated IL-10 expression. Together, these data suggest that microRNA-4661 can upregulate IL-10 and reduce the proliferation rate of PDLSCs.
\end{abstract}

Keywords: periodontitis; periodontal ligament stem cells; microRNA-4661; IL-10

\section{Introduction}

Periodontitis is not only a local lesion in mouth, but also a dysbiotic inflammatory disease that affects systemic health ${ }^{1}$. In the United States, periodontitis affects more than $50 \%$ of adults over the age of $30^{2}$. In China, the poor overall oral health conditions make periodontitis a substantial burden on public health ${ }^{3}$. It has been reported that the occurrence of periodontitis is correlated with multiple factors, including diabetes, being male, and having a low education level ${ }^{4}$. Treatment of periodontitis is most frequently accomplished using systemic antibiotics. However, the misuse of antibiotics, such as overdoses and improper drug selections, can lead to other serious and unfavorable health conditions, including drug resistance ${ }^{5}$. 
In the past several decades, studies have shown that the occurrence of periodontitis is also closely correlated with a set of genetic factors. Polymorphisms of IL-10 are correlated with a higher risk of periodontitis ${ }^{6}$. It is known that in periodontitis IL-10 can downregulate the synthesis of proinflammatory chemokines and cytokines, gelatinase, nitric oxide, and collagenase, thereby maintaining bone homeostasis ${ }^{7}$. It has also been reported that the development of periodontitis is usually accompanied with altered expression of microRNAs ${ }^{8}$, which are a group of small non-coding RNAs that play pivotal roles in RNA silencing and post-transcriptional regulation of gene expression?. MicroRNA-466l was first reported by high-throughput pyrosequencing in embryonic stem cells ${ }^{10}$. In some cases, microRNA-4661 requires interaction with IL-10 to achieve its biological functions ${ }^{10}$. The present study investigated the involvement of microRNA4661 and IL-10 in periodontitis.

\section{Methodology}

\section{Patients}

The inclusion and exclusion criteria, as well as the disease diagnostic standards, for the patients were based on the criteria set forth by Zheng et $\mathrm{al}^{11}$. All patients in this study ranged in age from 30 to 40 years old. Disease-free third molars and premolars were obtained from eight patients who were willing to receive orthodontic treatment. Diseased teeth were collected from another eight patients with generalized chronic periodontitis who were undergoing tooth extraction. The Ethics Committee of Nanjing Stomatological Hospital approved this study and all participants signed informed consent forms.

\section{Cell culture}

Explants of the periodontitis-affected and healthy periodontal tissues were collected from patients undergoing extraction and cultured. The middle part of the root was gently scraped to collect periodontal ligament (PDL) tissue. PDL tissues were cut into $1 \mathrm{~mm}^{3}$ cubes and transferred to a 6-well plate (Costar, Cambridge, USA) containing Minimum
Essential Medium (MEM, Sigma-Aldrich, St. Louis, USA) supplemented with $0.292 \mathrm{mg} / \mathrm{mL}$ glutamine (Sigma-Aldrich, St. Louis, USA), $100 \mu \mathrm{M}$ ascorbic acid (Sigma-Aldrich, St. Louis, USA), 100 U/mL penicillinstreptomycin (Sigma-Aldrich, St. Louis, USA), and $10 \%$ fetal bovine serum (FBS, Sigma-Aldrich, St. Louis, USA). Cells were grown at $37^{\circ} \mathrm{C}$ in a $5 \% \mathrm{CO}_{2}$ incubator. The isolation of PDLSCs was performed using the same method described in a previous study ${ }^{11}$. To separate PDLSCs, all cells were incubated with mouse anti-human monoclonal stem cell marker STRO-1 (1:100; R\&D Systems, Minneapolis, USA), and the STRO-1 ${ }^{+}$stem cells were isolated using immunomagnetic beads ${ }^{11}$.

\section{RNA isolation and RT-qPCR}

Total RNA was extracted using TRIzol reagent (Thermo Fisher Scientific, Waltham, USA). Extraction of microRNA was carried out using the TaqMan miRNA Isolation Kit (Applied Biosystems, Foster City, USA). Reverse transcription was performed according to the following reaction conditions: $50^{\circ} \mathrm{C}$ for $30 \mathrm{~min}$ and $85^{\circ} \mathrm{C}$ for $15 \mathrm{~min}$. RT-qPCR was performed to detect IL-10 mRNA and microRNA4661 using $\beta$-actin or U6 small nuclear RNA (snRNA) as endogenous controls. PCR reactions were performed on a StepOnePlus real-time PCR system (Applied Biosystems, Foster City, USA) with the following parameters: $95^{\circ} \mathrm{C}$ for $33 \mathrm{sec}$, followed by 40 cycles of $95^{\circ} \mathrm{C}$ for $13 \mathrm{sec}$ and $61^{\circ} \mathrm{C}$ for $23 \mathrm{sec}$. Each reaction was performed three times and $\mathrm{Ct}$ values were analyzed using $2^{-\Delta \Delta \mathrm{Ct}}$ method to calculate the relative expression levels of IL-10 mRNA and microRNA-4661. See Table 1 for sequences of primers used in RT-qPCR.

\section{Cell transfection}

The microRNA-4661 mimic and negative control microRNA were purchased as double-stranded RNA oligonucleotides from GenePharma (Shanghai, China). Mimics were transfected into PDLSCs at a dose of $10 \mathrm{nM}$. In this experiment, two cases of PDLSCs derived from both periodontitis-affected teeth and health teeth were used. Cells were incubated with microRNAs for $6 \mathrm{~h}$. Cells were harvested $24 \mathrm{~h}$ after transfection for experimental analysis. 
Table 1. The sequences of primers used in RT-qPCR analyses.

\begin{tabular}{lcc}
\hline Gene & Forward $\left(5^{\prime}-3^{\prime}\right)$ & Reverse $\left(5^{\prime}-3^{\prime}\right)$ \\
\hline IL-10 & GCCTAACATGCTTCGAGATC & TGATGTCTGGGTCTTGGTTC \\
$\beta$-actin & GACCTCTATGCCAACACAGT & AGTACTTGCGCTCAGGAGGA \\
microRNA-466I & ATGGCUAUAAAUACAUGCACA & GTGCAGGGTCCGAGGT \\
U6 & CTCGCTTCGGCAGCACA & AACGCTTCACGAATTTGCGT
\end{tabular}

\section{Cell proliferation assay}

PDLSCs were collected to make single cell suspensions at a density of $4 \times 10^{4}$ cells per well in a 96-well plate; PDLSCs from different patients were processed separately. A $0.1 \mathrm{~mL}$ cell suspension was added into each well of a 96-well plate. PDLSCs were cultured at $37^{\circ} \mathrm{C}$ with $5 \% \mathrm{CO}_{2}$. According to the manufacturer's instructions, $10 \mu \mathrm{L}$ of CCK- 8 solution (Sigma-Aldrich St. Louis, USA) was added into each well at $24,48,72$, and $96 \mathrm{~h}$ to measure dehydrogenase activity, which is proportional to the number of living cells. After incubation for $4 \mathrm{~h}$, optical density (OD) values were measured at $450 \mathrm{~nm}$.

\section{Western blot}

RIPA solution (Thermo Fisher Scientific, Waltham, USA) was added to the PDLSCs to extract total protein. Each well of a 10\% SDS-PAGE gel was loaded with $20 \mu \mathrm{g}$ of protein. After electrophoresis, we performed a gel transfer to a PVDF membrane and blocked by incubating with 5\% skim milk for $3 \mathrm{~h}$ at room temperature. Following the block, we incubated the membrane with primary antibodies against IL-10 (1:1,200, ab9969, Abcam, Cambridge, UK) or GAPDH (1:1,300, ab8245, Abcam, Cambridge, UK) overnight at $4^{\circ} \mathrm{C}$. ECL (Sigma-Aldrich, St. Louis, USA) was added to develop signals and a MYECL Imager (Thermo Fisher Scientific, Waltham, USA) was used to scan results. The gray values of the IL-10 bands were normalized to GAPDH using ImageJ software to measure the relative expression levels of IL-10.

\section{Statistical analysis}

SPSS19.0 (SPSS Inc., Chicago, USA) was used for all statistical analyses. Mean values were calculated from data of three biological replicates. A t-test (between two groups) or a one-way ANOVA and Tukey test (among multiple groups) was used for data comparison. $P<0.05$ was defined to indicate a statistically significant difference.

\section{Results}

\section{Decreased expression of microRNA-466I and IL-10 mRNA in PDLSCs derived from periodontitis-affected teeth}

The differential expression of genes in diseased lesions versus healthy tissues indicates the involvement of those genes in the disease. In this study, we detected the expression of microRNA-4661 and IL-10 mRNA in PDLSCs derived from periodontitis-affected teeth and healthy teeth using RT-qPCR. The expression levels of both microRNA-466l and IL-10 mRNA in PDLSCs derived from periodontitis-affected teeth were significantly lower than those derived from healthy teeth (Fig. 1, $P<0.05$ ).

\section{Increased rate of proliferation in PDLSCs derived from periodontitis-affected teeth}

The abnormal increase in the proliferation rate of PDLSCs is considered to be involved in the pathogenesis of periodontitis ${ }^{11}$. Therefore, we wanted to measure cell proliferation of PDLSCs derived from periodontitis-affected teeth and healthy teeth using CCK-8 assays. As shown in Fig. 2, the cell proliferation rate of the eight cases of PDLSCs derived from periodontitis-affected teeth was significantly higher than those derived from healthy teeth $(P<0.05)$.

\section{MicroRNA-466l overexpression upregulates IL-10 expression in PDLSCs derived from periodontitis-affected teeth and healthy teeth}

The IL-10 anti-inflammatory pathway is involved in periodontitis, and it is known that microRNA-4661 sometimes requires interaction with IL-10 to achieve 
A

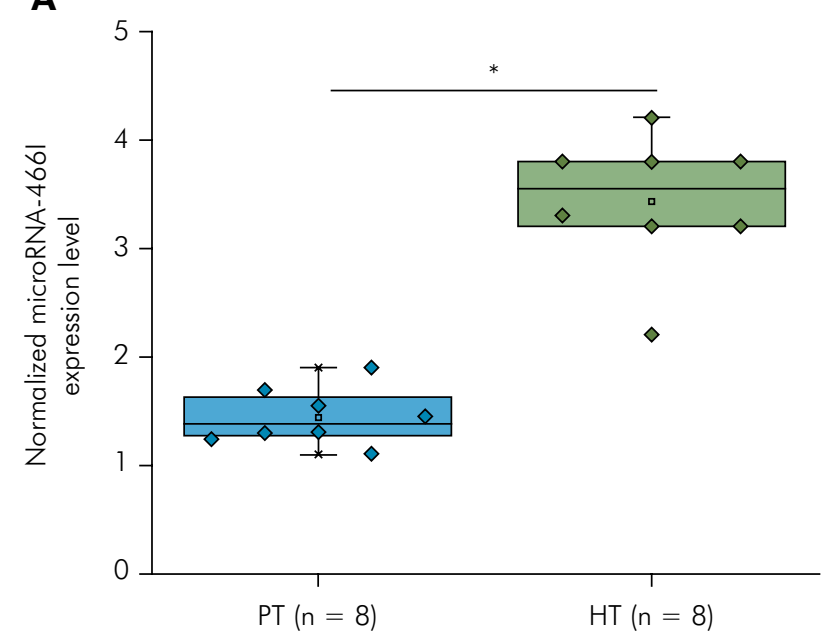

B

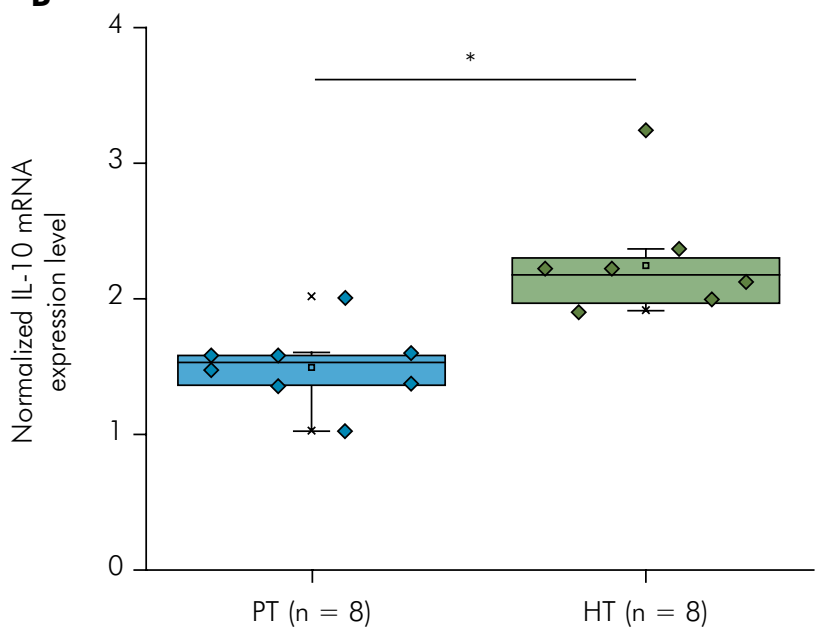

Notes: ${ }^{*}, P<0.05 ; \mathrm{PT}$, periodontitis-affected teeth; $\mathrm{HT}$, healthy teeth.

Figure 1. Expression of microRNA-466I (a) and IL-10 mRNA (b) in PDLSCs derived from periodontitis-affected teeth or healthy teeth. This figure shows thet expression of microRNA-466I (a) and IL-10 mRNA (b) in PDLSCs derived from periodontitis-affected teeth and health teeth.

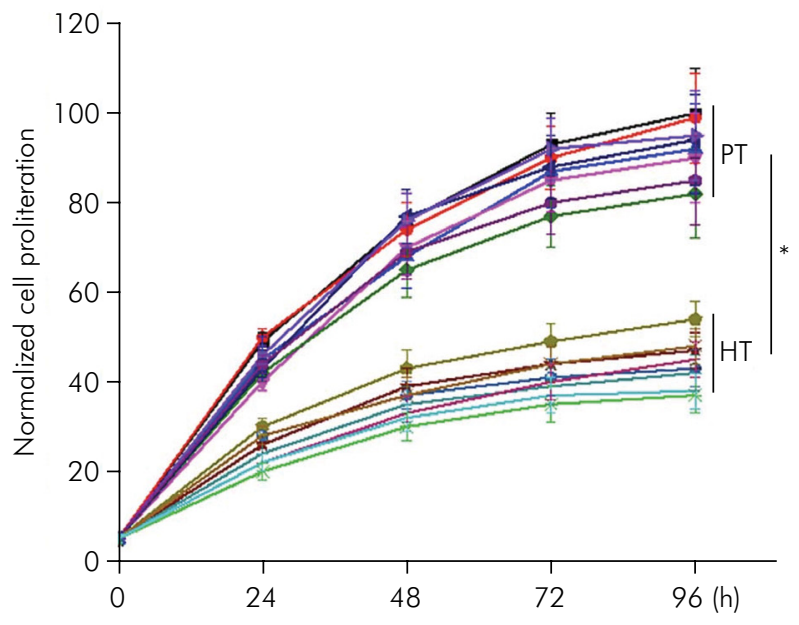

Notes: ${ }^{*}, P<0.05$; PT, periodontitis-affected teeth; $\mathrm{HT}$, healthy teeth.

Figure 2. Proliferation of PDLSCs derived from periodontitisaffected teeth or healthy teeth.

its biological roles ${ }^{10}$. In this study, a microRNA-4661 mimic was transfected into two different cultures of PDLSCs derived from periodontitis-affected teeth and two different cultures of PDLSCs derived from healthy teeth. As shown in Fig. 3, transfection of the microRNA-4661 mimic significantly upregulates the expression of IL-10 in both cultures of PDLSCs derived from periodontitis-affected teeth and both cultures of PDLSCs derived from healthy teeth $(P<0.05)$.

\section{Effects of microRNA-466I overexpression on proliferation of PDLSCs derived from periodontitis-affected teeth and health teeth}

We performed CCK-8 assays to investigate the impact of microRNA-4661 overexpression on the proliferation of PDLSCs derived from periodontitisaffected teeth and healthy teeth. As shown in Fig. 4, microRNA-466l overexpression resulted in significantly reduced proliferation rates in PDLSCs derived from periodontitis-affected teeth (Fig. 4a), as well as healthy teeth (Fig. $4 b)(P<0.05)$. It is worth noting that PDLSCs derived from other cases of periodontitis-affected teeth ( $n=3$, Fig. S1A) and healthy teeth ( $n=1$, Fig. S1B) were tested and similar results were obtained.

\section{Discussion}

The key finding of our study is that microRNA-4661 potentially plays a pivotal role in inhibiting the antiviral innate immune response $\mathrm{e}^{12}$ and may also 
A

$$
\text { C }
$$

IL-10

GAPDH

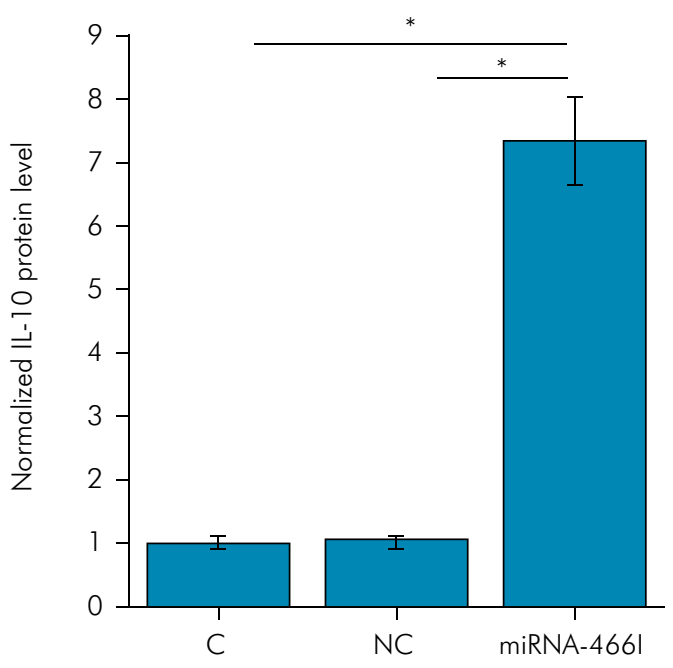

B
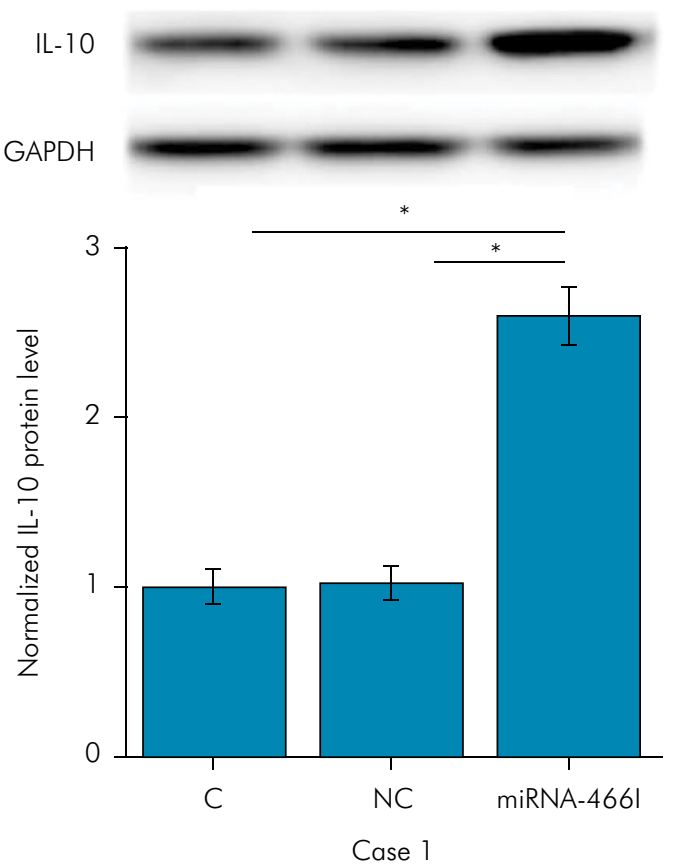

Case 2
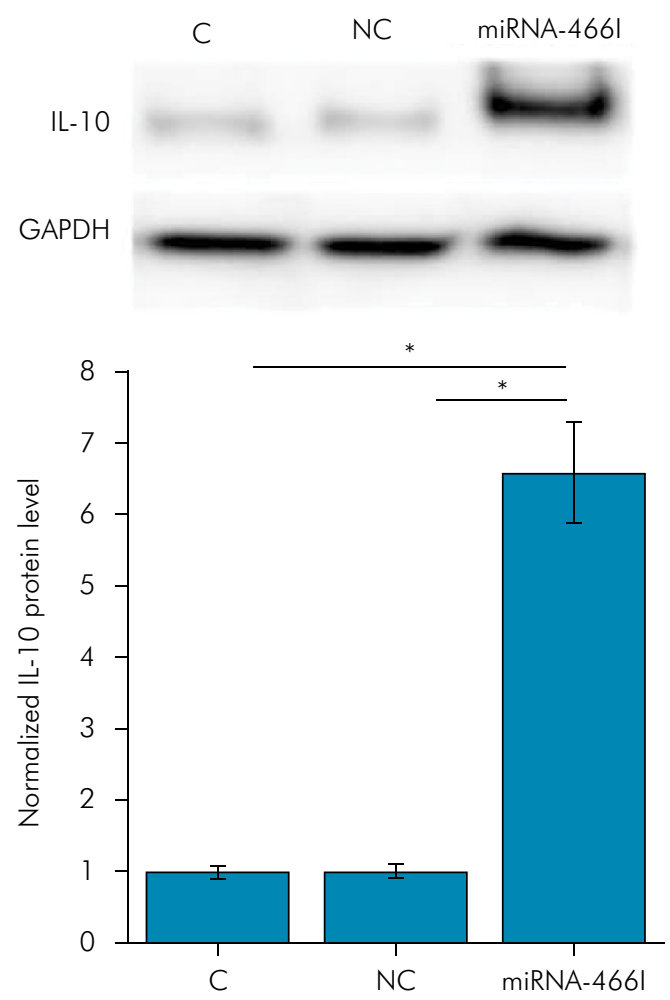

Case 2

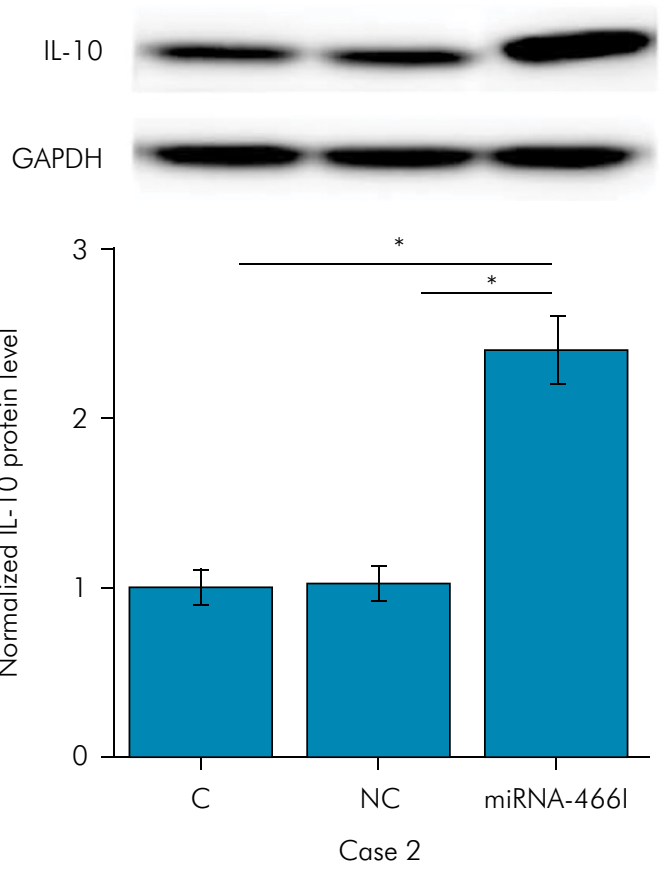

Notes: ${ }^{*}, P<0.05 ; C$, control cells without transfection; NC, cells transfected with the negative control microRNA mimic.

Figure 3. Effects of microRNA-466l overexpression on IL-10 expression in two cultures of PDLSCs derived from periodontitis-affected teeth (a) and two cultures of PDLSCs derived from healthy teeth (b). 
A
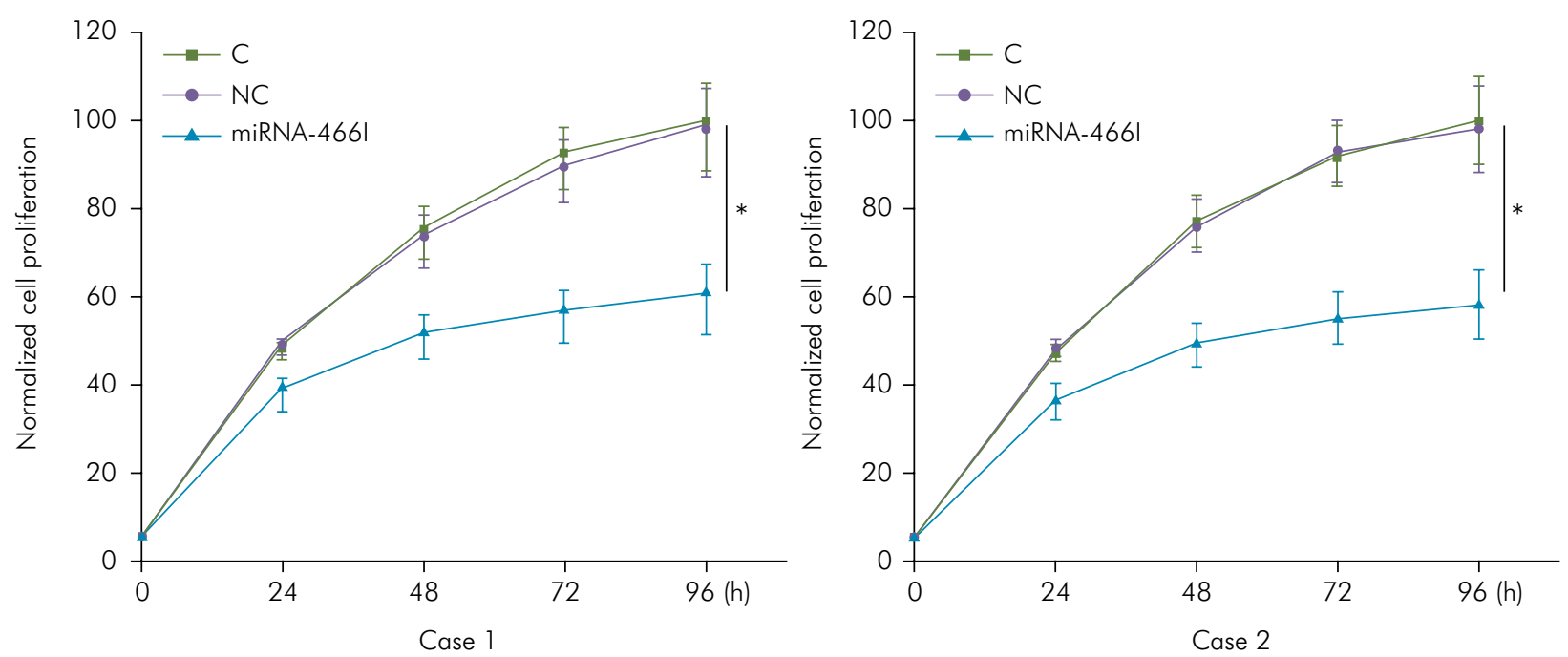

B
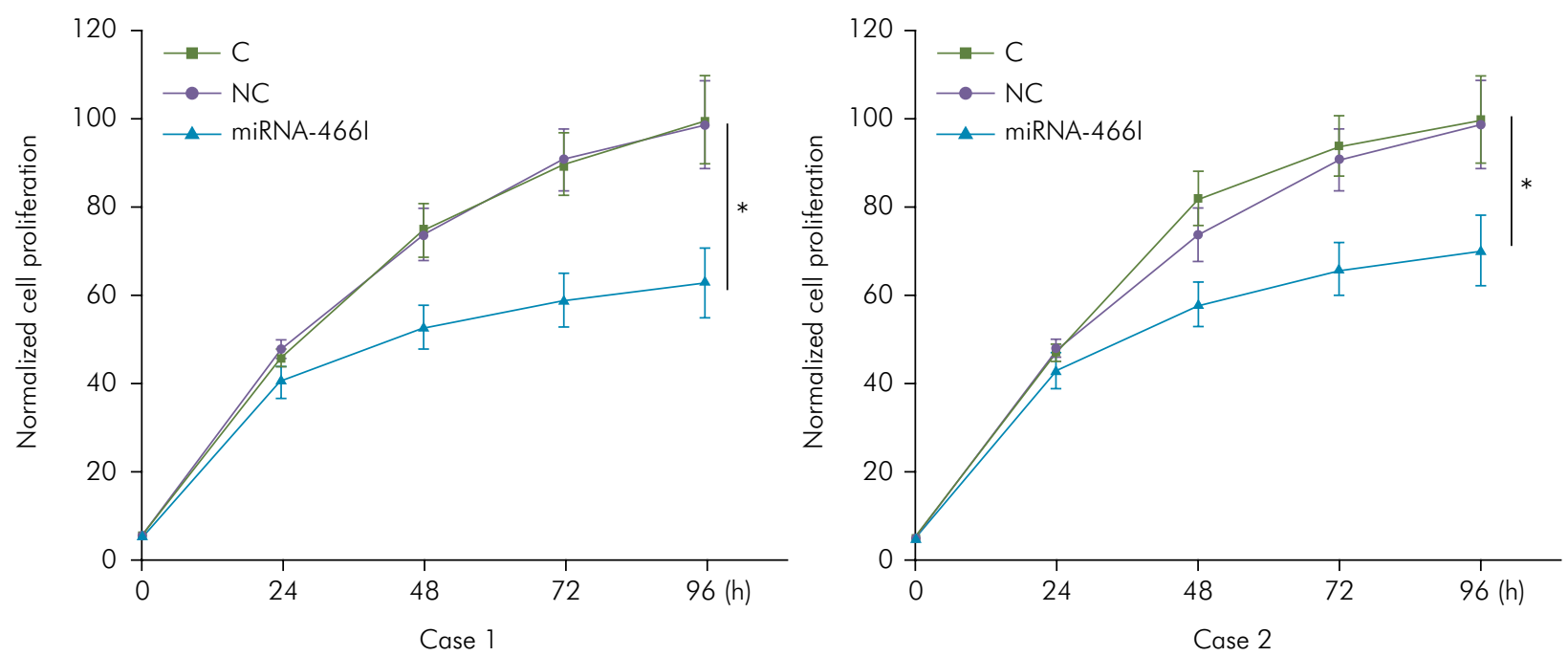

Notes: ${ }^{*}, P<0.05 ; C$, control cells without transfection; NC, cells transfected with the negative control microRNA mimic.

Figure 4. Effects of microRNA-466l overexpression on the proliferation of two cultures of PDLSCs derived from periodontitis-affected teeth (a) and two cultures of PDLSCs derived from healthy teeth (b).

inhibit the development of periodontitis, which is a disease related to innate antiviral immunity ${ }^{13}$. We also observed that the role of microRNA-4661 in inhibiting periodontitis is likely achieved by reducing the proliferation rate of PDLSCs, which is a common occurrence in periodontitis-affected tissues.

By comparing the genome-wide microRNA expression in healthy and periodontitis-affected tissues, a large set of differential microRNA expression has been identified ${ }^{8}$, indicating the involvement of microRNAs in this disease. The specific functionality of microRNAs in periodontitis pathogenesis remains largely unexplored, except for a recent study demonstrating that miR-1226 in gingival crevicular fluid can serve as a potential biomarker for periodontitis ${ }^{14}$. However, the molecular mechanism of the action that relates miR-1226 to periodontitis is still unspecified. It is known that the 
A
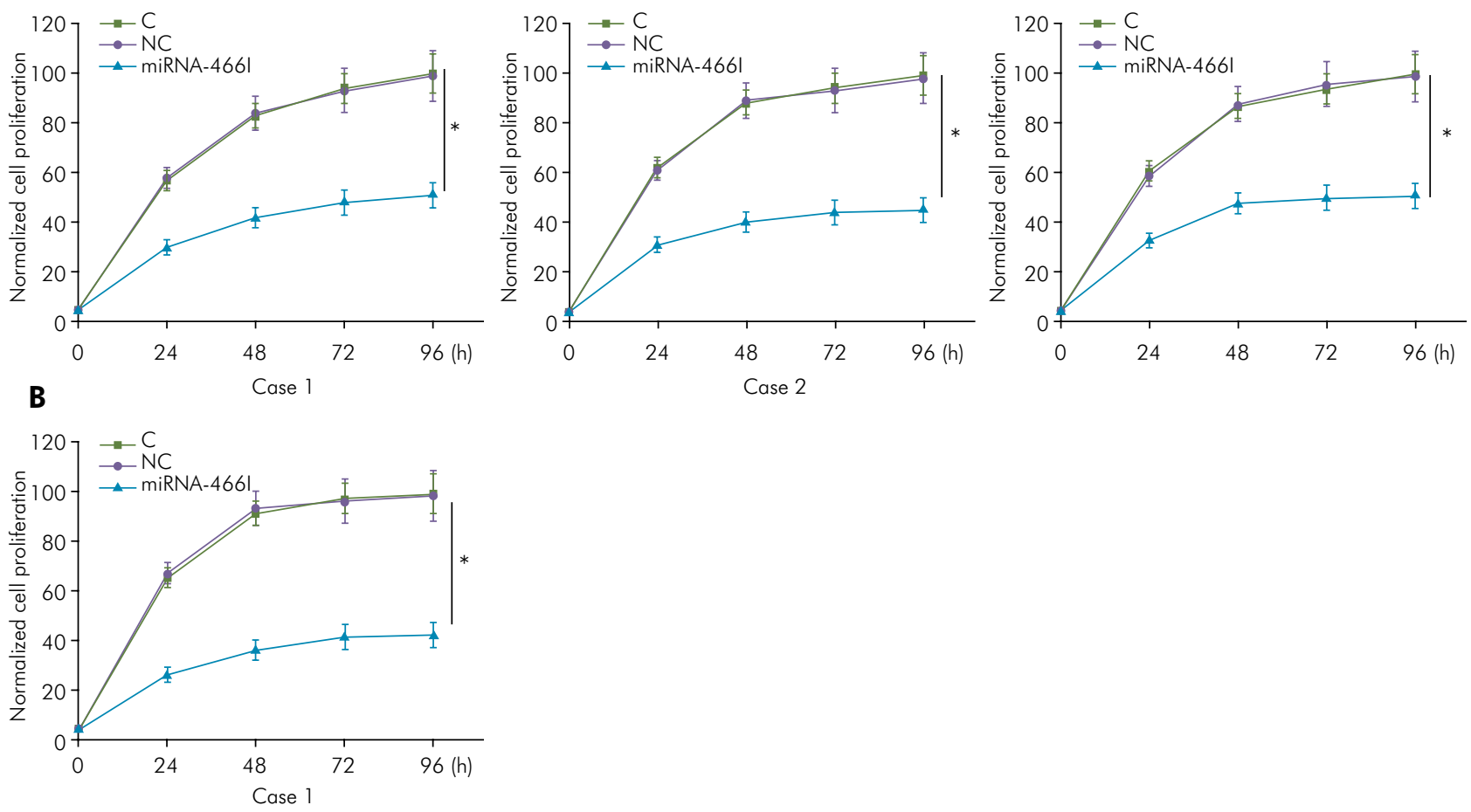

Notes: *, $P<0.05$; $C$, control cells without transfection; NC, cells transfected with the negative control microRNA mimic.

Figure S1. Effects of microRNA-466l overexpression on the proliferation of three cultures of PDLSCs derived from periodontitisaffected teeth (a) and one culture of PDLSCs derived from healthy teeth (b).

development of periodontitis is closely correlated with altered innate antiviral immunity ${ }^{13}$, a state in which microRNA-4661 plays a pivotal role ${ }^{12}$. In our study, the expression of microRNA-4661 was found to be significantly downregulated in PDLSCs derived from periodontitis-affected teeth compared to PDLSCs derived from healthy teeth, indicating that the downregulation of microRNA-4661 expression was likely involved in the pathogenesis of periodontitis.

Periodontitis is essentially a type of inflammatory disease that requires the involvement of multiple anti- or pro-inflammatory factors, such as IL-1, IL-6, and IL-10 ${ }^{15,16}$. Many studies have shown that IL-10 is likely to be the most important element among those factors in periodontitis ${ }^{6,7}$, and the inhibition of IL-10 signaling characterizes this disease. In our study, the expression levels of IL-10 were found to be significantly reduced in PDLSCs derived from periodontitis-affected teeth compared to PDLSCs derived from healthy teeth, further confirming the involvement of IL-10 signal inhibition in this disease. The interactions between microRNAs and IL-10 play critical roles in different pathological changes or physiological processes ${ }^{17}$. It has been reported that microRNA-4661 is an upstream activator of IL-10 expression in certain immune responses ${ }^{10}$. In our study, microRNA-466l overexpression significantly promoted the expression of IL-10 in PDLSC cultures derived from both periodontitis-affected teeth and healthy teeth. It is also worth noting that the upregulation of IL-10 was more significant in PDLSCs derived from periodontitis-affected teeth than in PDLSCs derived from healthy teeth, which is possibly due to the low basal expression level of IL-10 in PDLSCs derived from periodontitisaffected teeth.

The abnormal increase in the proliferation rate of human PDLSCs is considered to be involved in the pathogenesis of periodontitis ${ }^{11}$, and the inhibition of 
PDLSC proliferation has been used as a therapeutic target for the treatment of periodontitis ${ }^{18,19}$. In our study, proliferation rates of all eight cases of human PDLSCs derived from periodontitis-affected teeth were significantly higher than in PDLSCs derived from healthy teeth, further confirming the existence of accelerated PDLSC proliferation in periodontitis. Our study also showed that microRNA-466l overexpression significantly reduced the proliferation rate of PDLSCs derived from both periodontitis-affected teeth and healthy teeth. Therefore, microRNA-466l might serve as potential therapeutic target for periodontitis, although the effect on normal PDLSCs will need to be considered.

\section{Conclusions}

MicroRNA-4661 was observed to be downregulated in PDLSCs derived from periodontitis-affected teeth compared to PDLSCs derived from healthy teeth. MicroRNA-4661 potentially inhibits periodontitis by upregulating IL-10 expression and reducing the proliferation rate of PDLSCs.

\section{Acknowledgments}

We would like to thank the financial support from the National Natural Science Foundation of China (51472115, 51772144) and the Nanjing Medical Science and Technique Development Foundation (QRX17080).

\section{References}

1. Hajishengallis G. Periodontitis: from microbial immune subversion to systemic inflammation. Nat Rev Immunol. 2015;15(1):30-44.

2. Eke PI, Dye BA, Wei L, et al. Update on prevalence of periodontitis in adults in the United States: NHANES 2009 to 2012. J Periodontol. 2015;86(5):611-22

3. Zhang Q, Li Z, Wang C, et al. Prevalence and predictors for periodontitis among adults in China, 2010. Glob Health Action. 2014;7(1):24503.

4. Van Dyke TE, Dave S. Risk factors for periodontitis. J Int Acad Periodontol. 2005;7(1):3-7.

5. Cionca N. Use and misuse of systemic antibiotics in periodontitis treatment. Oral Health Prev Dent. 2017;15(4):305-6.

6. Yang SL, Huang SJ. Interleukin-10 polymorphisms (rs1800871, rs1800872 and rs1800896) and periodontitis risk: A meta-analysis. Arch Oral Biol. 2019;97:59-66.

7. Graunaite I, Lodiene G, Maciulskiene V. Pathogenesis of apical periodontitis: a literature review. J Oral Maxillofac Res. 2011;2(4):e1.

8. Lee W, Young H, Am Na HS, et al. Comparison of inflammatory microRNA expression in healthy and periodontitis tissues. Biocell. 2011;35(2):43-9.

9. Bartel DP. MicroRNAs: genomics, biogenesis, mechanism, and function. Cell. 2004;116(2):281-97.

10. Ma F, Liu X, Li D, et al. MicroRNA-466l upregulates IL-10 expression in TLR-triggered macrophages by antagonizing RNA-binding protein tristetraprolin-mediated IL-10 mRNA degradation. J Immunol. 2010;184(11):6053-9.

11. Zheng W, Wang S, Wang J, et al. Periodontitis promotes the proliferation and suppresses the differentiation potential of human periodontal ligament stem cells. Int J Mol Med. 2015;36(4):915-22.

12. Li Y, Fan X, He X, et al. MicroRNA-466l inhibits antiviral innate immune response by targeting interferon-alpha. Cell Mol Immunol. 2012;9(6):497-502.

13. Mahanonda R, Sa-Ard-lam N, Rerkyen P, et al. Innate antiviral immunity of periodontal tissue. Periodontology. 2000 2011;56(1):143-53.

14. Micó-Martínez P, García-Giménez JL, Seco-Cervera M, et al. miR-1226 detection in GCF as potential biomarker of chronic periodontitis: A pilot study. Med Oral Patol Oral Cir Bucal. 2018;23(3):e308-14.

15. Hajishengallis G. Dysbiosis and inflammation in periodontitis: synergism and implications for treatment. Journal of Oral Microbiology. 2017;9(supl):1325198.

16. Reis C, Da Costa AV, Guimarães JT, et al. Clinical improvement following therapy for periodontitis: Association with a decrease in IL-1 and IL-6. Exp Ther Med. 2014;8(1):323-7.

17. McCoy CE, Sheedy FJ, Qualls JE, et al. IL-10 inhibits miR-155 induction by toll-like receptors. J Biol Chem. 2010;285(27):20492-8.

18. Liu Y, Zheng Y, Ding G, et al. Periodontal ligament stem cell-mediated treatment for periodontitis in miniature swine. Stem Cells. 2008;26(4):1065-73.

19. Ding G, Liu Y, Wang W, et al. Allogeneic periodontal ligament stem cell therapy for periodontitis in swine. Stem Cells. 2010;28(10):1829-38 


\section{Upregulation of IL-10 expression inhibits the proliferation of human periodontal ligament stem cells. Braz Oral Res. 2020;34e030.}

Affiliation

Where is read:

Nanjing University, Medical School, Department of Periodontology, Nanjing City, Jiangsu Province, China.

It should read:

Nanjing Stomatological Hospital, Department of Periodontology, Nanjing City, Jiangsu Province. China. 